\title{
Microwave Assisted Synthesis, Antifungal Activity, and DFT Study of Some Novel Triazolinone Derivatives
}

\author{
Na-Bo Sun, Jian-Zhong Jin, and Fang-Yue He \\ College of Biology and Environmental Engineering, Zhejiang Shuren University, Hangzhou, Zhejiang 310015, China \\ Correspondence should be addressed to Na-Bo Sun; nabosun@gmail.com
}

Received 25 December 2014; Revised 5 February 2015; Accepted 15 February 2015

Academic Editor: Lucia Lopalco

Copyright ( $) 2015 \mathrm{Na}$-Bo Sun et al. This is an open access article distributed under the Creative Commons Attribution License, which permits unrestricted use, distribution, and reproduction in any medium, provided the original work is properly cited.

\begin{abstract}
A series of some novel 1,2,4-triazol-5(4H)-one derivatives were designed and synthesized under microwave irradiation via multistep reaction. The structures of 1,2,4-triazoles were confirmed by ${ }^{1} \mathrm{H}$ NMR, MS, FTIR, and elemental analysis. The antifungal activities of 1,2,4-triazoles were determined. The antifungal activity results indicated that the compounds $\mathbf{5 c}$, $\mathbf{5} \mathbf{f}$, and $\mathbf{5 h}$ exhibited good activity against Pythium ultimum, and the compounds $\mathbf{5 b}$ and $\mathbf{5} \mathbf{c}$ displayed good activity against Corynespora cassiicola. Theoretical calculation of the compound $\mathbf{5 c}$ was carried out with B3LYP/6-31G (d). The full geometry optimization was carried out using 6$31 \mathrm{G}(\mathrm{d})$ basis set, and the frontier orbital energy and electrostatic potential were discussed, and the structure-activity relationship was also studied.
\end{abstract}

\section{Introduction}

Nowadays, nitrogen-containing heterocycles became a research hot spot because they displayed excellent activities [1-9]. 1,2,4-Triazole derivatives, especially triazolinone compounds, exhibited diverse activities, such as the commercial antidepressant medicine Trazodone, herbicide azafenidin, and herbicides amicarbazone, sulfentrazone, and carfentrazoneethyl. So the synthesis of substituted triazolinone compounds is one of the important fields for many researchers. Many references reported that triazolinone derivatives showed other interesting activities, including angiotensin II AT(1) receptor antagonists [10-12], anti-human immunodeficiency virus (HIV) activity [13, 14], acetolactate synthase (ALS) inhibitors $[15,16]$, protoporphyrinogen oxidase inhibitors [17], antioxidant activities [18-20], anticancer activity [21, 22], and anti-inflammatory activity [23].

Microwave-assisted technique is a green method in current organic synthesis [24-28]. It is attractive, offering reduced pollution, low cost, and high yields. The green technique can often shorten the reaction time.

In our previous work [29-33], some 1,2,4-triazole compounds were designed and synthesized. They showed good antifungal activities. In this paper, fifteen novel 1,2,4-triazole derivatives were designed and synthesized under microwave irradiation. Their chemical structures were confirmed by ${ }^{1}$ H NMR, FTIR, MS, and elemental analysis. The antifungal activity of 1,2,4-triazoles was determined in vivo.

\section{Results and Discussion}

2.1. Synthesis. The synthetic route of target compounds was outlined in Scheme 1. All the reported syntheses of O-methyl carbonisothiocyanatidate involve reaction of a thiocyanate salt (e.g., $\mathrm{Pb}^{2+}, \mathrm{NH}_{4}^{+}, \mathrm{K}^{+}$, and $\mathrm{Na}^{+}$) with methyl carbonochloridate. If we use equal molar amounts of potassium thiocyanate and methyl carbonochloridate, approximately equal amounts of both isomers will be obtained. When the methyl carbonochloridate was reacted with potassium thiocyanate, the potassium thiocyanate was excess, while the drop speed must be slow, as fast speed decreased the yield of product. The intermediate 2 was easily prepared by the reaction of methoxycarbonyl isothiocyanate and methanol. In the synthesis process of intermediate $\mathbf{3}$, the intermediate $\mathbf{2}$ cyclized with hydrazine hydrate. Because it is an equilibrium reaction it is reacted under nitrogen atmosphere in order to off hydrogen sulfide gas and increase the yield of intermediate 3. The intermediate $\mathbf{3}$ exhibits two $\mathrm{NH}$ groups, which may 


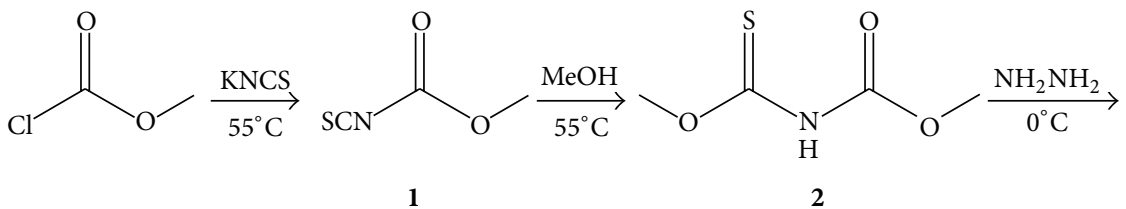

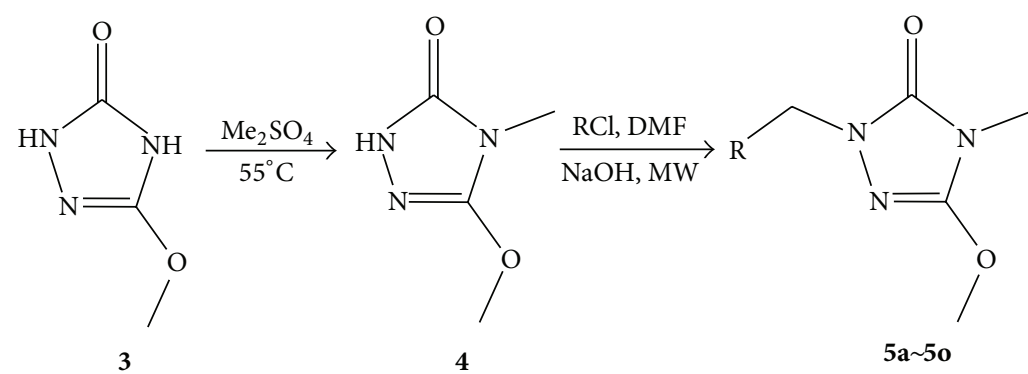

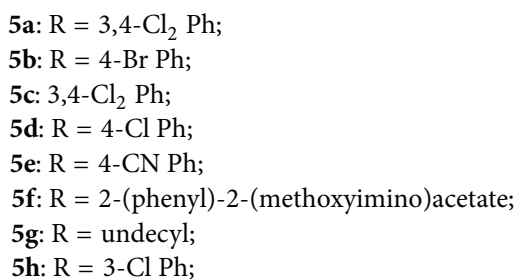

5i: $\mathrm{R}=2-\mathrm{Cl} \mathrm{Ph}$;

5j: $\mathrm{R}=3-\mathrm{CN} \mathrm{Ph}$;

5k: $\mathrm{R}=4-\mathrm{MeO} \mathrm{Ph}$;

5l: $\mathrm{R}=3$-F Ph;

5m: $\mathrm{R}=4-\mathrm{F} \mathrm{Ph}$

5n: $\mathrm{R}=5$-chloropyridin-2-yl;

5o: $\mathrm{R}=2-\mathrm{FPh}$

SCHEme 1: The synthetic route of title compounds.

be both methylated with $\left(\mathrm{CH}_{3}\right)_{2} \mathrm{SO}_{4}$. We found that the $\mathrm{pH}$ values of two $\mathrm{NH}$ groups are different. Therefore, $\mathrm{pH}$ value is controlled preferably about 8 to 9 which is given the intermediate 4 . The target compounds $\mathbf{5 a} \sim \mathbf{5 o}$ were synthesized using microwave irradiation method. The signal of $\mathrm{NCH}_{2}$ proton appeared around $\delta 3.66-5.04 \mathrm{ppm}$. The infrared spectrum of the title compounds $\mathbf{5 a} \sim \mathbf{5 o}$ showed absorption bands around $2930 \mathrm{~cm}^{-1}$ for $\mathrm{CH}_{2}$ stretching. The characteristic stretching vibration $v(\mathrm{C}=\mathrm{O})$ appears at $1720 \mathrm{~cm}^{-1}$. The mass spectrum results showed that molecular ion is in accordance with its molecular formula. The elemental analysis results are in accordance with the calculated results.

2.2. Antifungal Activity. The in vivo antifungal activity results of 1,2,4-triazol-5(4H)-ones against Phytophthora infestans, Botrytis cinerea, Corynespora cassiicola, Rhizoctonia solani, and Pythium ultimum were shown in Table 1; dimethomorph, fludioxonil, chlorothalonil, validamycin, and zhongshengmycin were used as controls. From Table 1, it is shown that compounds $\mathbf{5 c}, \mathbf{5} \mathbf{f}$, and $\mathbf{5 h}$ exhibited good control efficacy against Pythium ultimum at $500 \mathrm{ppm}$. Compounds 5a, 5g, $5 \mathbf{i}, \mathbf{5 m}$, and $5 \mathrm{n}$ showed moderate control efficacy against Pythium ultimum. The control zhongshengmycin had no control efficacy against Pythium ultimum. For the Rhizoctonia solani, most of the title compounds displayed no control efficacy, except compounds $\mathbf{5} \mathbf{f}$ and $\mathbf{5 h}$. Surprisingly, all the compounds can not only inhibit the Botrytis cinerea, but also promote the Botrytis cinerea growth. Most of 1,2,4triazol-5(4H)-ones displayed weak control efficacy against Corynespora cassiicola; only compounds $\mathbf{5 b}$ and $\mathbf{5 c}$ showed good antifungal activity (about 70\%) against Corynespora cassiicola, which is higher than that of control chlorothalonil. Unfortunately, the title compounds exhibited weak activity against Phytophthora infestans.

2.3. DFT Calculation. Molecular total energy and frontier orbital energy levels are listed in Table 2. Energy gap between HOMO and LUMO was calculated by B3LYP.

According to the frontier molecular orbital theory, HOMO and LUMO are the most important factors that affect the bioactivity. HOMO has the priority to provide electrons, while LUMO can accept electrons firstly [34-36]. Thus, study on the frontier orbital energy can provide useful information about the biological mechanism. From Figure 1, the HOMO of compound $\mathbf{5 c}$ is mainly located on the $\mathrm{OCH}_{3}$ group and 1,2,4-triazol-5(4H)-one ring, while the LUMO of compound $5 \mathbf{c}$ is located on the $\mathrm{OCH}_{3}$ group, 2,4- $\mathrm{Cl}_{2}$ benzene ring, and 1,2,4-triazol-5(4H)-one ring. The fact that the compound 5c has strong affinity suggests the importance of the frontier molecular orbital in the $\pi-\pi$ stacking or hydrophobic interactions. From Figure 1, the electron transfer process of the HOMO and LUMO implies that $2,4-\mathrm{Cl}_{2}$ phenyl ring had important impact on the antifungal activity.

The electrostatic potential of compound $\mathbf{5 c}$ was also calculated. From Figure 2, it is clear that the oxygen atom at the 1,2,4-triazole ring possessed the greatest negative charges and it is therefore possible that the oxygen atom had some interaction with the receptor or acceptor.

Furthermore, the combination of MO provided meaningful clues as to the structural features of these new family fungicides that will be helpful in the design of more potent compounds in the future. 


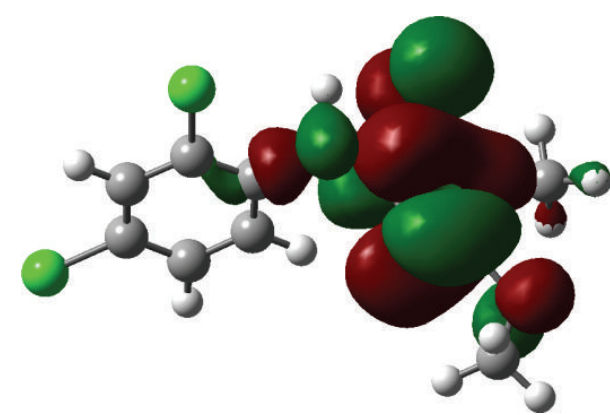

(a)

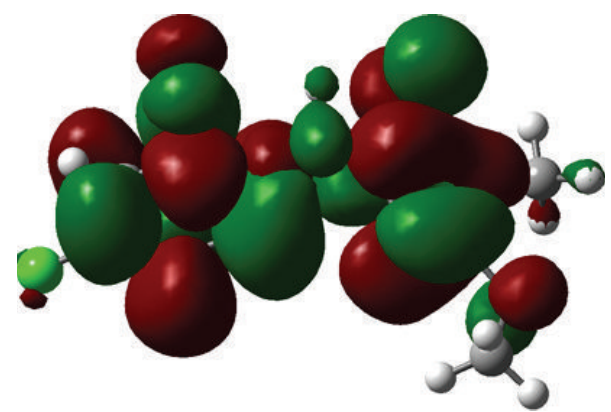

(b)

FIGURE 1: Frontier molecular orbitals of compound 5c: (a) LUMO of compound 5c; (b) HOMO of compound 5c.

TABLE 1: The antifungal activity of title compounds in vivo at $500 \mathrm{ppm}(\%)$.

\begin{tabular}{|c|c|c|c|c|c|}
\hline Number & Phytophthora infestans & Botrytis cinerea & Corynespora cassiicola & Rhizoctonia solani & Pythium ultimum \\
\hline $5 a$ & 25.66 & -37.95 & 19.81 & 0 & 44.44 \\
\hline $5 b$ & -0.8 & -61.1 & 68.6 & 3.33 & -11.11 \\
\hline $5 c$ & -0.8 & -61.1 & 73.93 & 0 & 77.78 \\
\hline $5 d$ & -0.8 & -49.52 & 26.26 & 0 & 11.11 \\
\hline $5 e$ & -0.8 & -38.91 & 13.08 & 0 & -88.89 \\
\hline $5 f$ & -0.8 & -51.45 & 18.13 & 33.89 & 88.89 \\
\hline $5 \mathrm{~g}$ & -0.8 & -56.28 & 9.72 & 2.22 & 33.33 \\
\hline $5 \mathrm{~h}$ & -0.8 & -52.42 & 6.36 & 29.44 & 66.67 \\
\hline $5 i$ & -0.8 & -38.91 & 37.76 & 0 & 44.44 \\
\hline $5 \mathbf{j}$ & -0.8 & -48.56 & 39.44 & 0 & -11.11 \\
\hline $5 k$ & -0.8 & -33.12 & 34.95 & 0 & -22.22 \\
\hline 51 & 29.16 & -27.34 & 37.76 & 0 & -22.22 \\
\hline $5 \mathrm{~m}$ & -0.8 & -7.08 & 24.86 & 0 & 33.33 \\
\hline $5 n$ & -0.8 & -42.77 & 0.19 & 11.11 & 33.33 \\
\hline 50 & -0.8 & -20.58 & 4.11 & 0 & 11.11 \\
\hline Dimethomorph & 97.76 & & & & \\
\hline Fludioxonil & & 86.98 & & & \\
\hline Chlorothalonil & & & 45.89 & & \\
\hline Validamycin & & & & 62.50 & \\
\hline Zhongshengmycin & & & & & 0 \\
\hline
\end{tabular}

TABLE 2: Total energy and frontier orbital energy.

\begin{tabular}{lc}
\hline & DFT \\
\hline$E_{\text {total }} /$ Hartree $^{\mathrm{b}}$ & -1660.90340104 \\
$E_{\mathrm{HOMO}} /$ Hartree & -0.21696 \\
$E_{\mathrm{LUMO}} /$ Hartree & -0.02347 \\
$\Delta E^{\mathrm{a}} /$ Hartree & 0.19349 \\
\hline
\end{tabular}

${ }^{\mathrm{a}} \Delta E=E_{\mathrm{LUMO}}-E_{\mathrm{HOMO}}$.

${ }^{\mathrm{b}} 1$ Hartree $=4.35974417 \times 10^{-18} \mathrm{~J}=27.2113845 \mathrm{ev}$.

\section{Materials and Methods}

3.1. Instruments. Melting points were measured using an $\mathrm{X}-4$ melting apparatus and were uncorrected. ${ }^{1} \mathrm{H}$ NMR spectra were determined on a Bruker AC-P400 instrument $(400 \mathrm{MHz})$ using TMS as an internal standard and $\mathrm{CDCl}_{3}$ as solvent. Mass spectra were determined on a Thermo Finnigan
LCQ Advantage LC/mass detector instrument. Elemental analyses were recorded on a Yanaco MT-3CHN elemental analyzer. Microwave activation was carried out with CEM Discover Focused Microwave $(2450 \mathrm{MHz}, 300 \mathrm{~W})$. All the reagents are of analytical grade or freshly prepared before use. The course of the reactions was monitored by TLC; analytical TLC was performed on silica gel GF 254.

3.2. Synthetic Procedures. The synthetic route is shown in Scheme 1.

3.2.1. Synthesis of Intermediates 1 and 2. The potassium thiocyanate $(10.69 \mathrm{~g}, 0.11 \mathrm{~mol})$ and pyridine $(0.40 \mathrm{~g})$ were dissolved in methyl isobutyl ketone $(50 \mathrm{~mL})$; methyl chloroformate $(9.45 \mathrm{~g}, 0.10 \mathrm{~mol})$ was added dropwise at $55^{\circ} \mathrm{C}$, and the mixture was stirred for $4 \mathrm{~h}$. Then $\mathrm{MeOH}(20 \mathrm{~mL})$ was added to the mixture and stirred for $16 \mathrm{~h}$. The mixture was washed with concentrated hydrochloric acid $(3 \mathrm{~mL})$ and $\mathrm{H}_{2} \mathrm{O}$ 

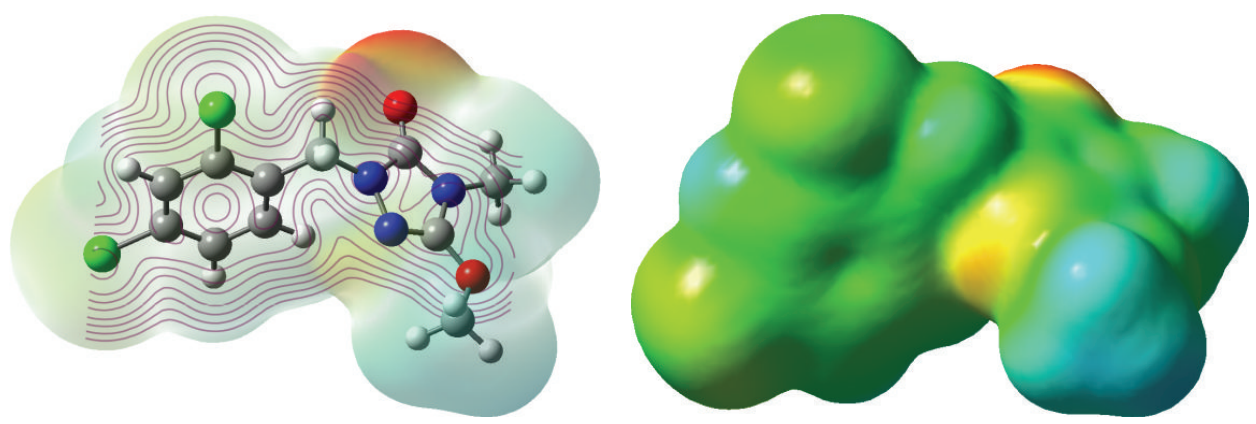

FIgURE 2: The ESP of compound 5c.

$(50 \mathrm{~mL})$. After filtration and evaporation of the solvent, the crude intermediate 2 was collected without further purification: white solid, yield $80 \%,{ }^{1} \mathrm{H} \mathrm{NMR}\left(400 \mathrm{MHz} \mathrm{CDCl}_{3}\right) \delta$ : 3.77 (s, 3H, $\mathrm{COOCH}_{3}$ ), 4.12 (s, $\left.3 \mathrm{H}, \mathrm{CSOCH}_{3}\right), 8.56$ (s, $1 \mathrm{H}$, $\mathrm{NH})$.

3.2.2. Synthesis of Intermediate 3. To a solution of intermediate $2(50 \mathrm{mmol})$ in $\mathrm{MeOH}(75 \mathrm{~mL})$ were added $80 \%$ $\mathrm{NH}_{2} \mathrm{NH}_{2} \cdot \mathrm{H}_{2} \mathrm{O}(4.07 \mathrm{~g}, 65 \mathrm{mmol})$ and $\mathrm{KOH}(45 \%, 0.81 \mathrm{~g}$, $6.5 \mathrm{mmol}$ ) at $0^{\circ} \mathrm{C}$; then the mixture was stirred at $30^{\circ} \mathrm{C}$ for $5 \mathrm{~h}$. After evaporation of the solvent, the crude intermediate 3 was recrystallized by EtOH to give white crystal 3: yield 78\%, m.p. $172 \sim 173^{\circ} \mathrm{C},{ }^{1} \mathrm{H}$ NMR (400 MHz, DMSO- $d_{6}$ ) $\delta: 4.02$ (s, $3 \mathrm{H}$, triazolone- $\left.\mathrm{OCH}_{3}\right), 10.51(\mathrm{~s}, 1 \mathrm{H}, \mathrm{NH})$.

3.2.3. Synthesis of Intermediate 4. To a solution of 3 $(65 \mathrm{mmol})$ and $\mathrm{K}_{2} \mathrm{CO}_{3}(9.52 \mathrm{~g})$ in $\mathrm{CH}_{3} \mathrm{CN}(100 \mathrm{~mL})$ was added $\left(\mathrm{CH}_{3}\right)_{2} \mathrm{SO}_{4}(68 \mathrm{mmol})$ at $55^{\circ} \mathrm{C}$, and the mixture was stirred for another $2 \mathrm{~h}$. The organic phase was extracted with $\mathrm{CH}_{2} \mathrm{Cl}_{2}(3 \times 10 \mathrm{~mL})$. After drying over sodium sulphate and evaporation of the solvent, the crude was collected without being purified to give the corresponding intermediate 4 .

3.2.4. General Procedure for Thioether 5. DMF ( $5 \mathrm{~mL}), 4$ (0.25 g, $1.00 \mathrm{mmol}), \mathrm{RCH}_{2} \mathrm{Cl}(1.10 \mathrm{mmol})$, and $\mathrm{NaOH}(0.05 \mathrm{~g}$, $1.20 \mathrm{mmol}$ ) were charged into a CEM designed $10 \mathrm{~mL}$ pressure-rated vial. Then it was irradiated in a CEM Discover Focused Synthesizer ( $150 \mathrm{w}, 90^{\circ} \mathrm{C}, 200 \mathrm{psi}, 15$ minutes). The mixture was cooled below $50^{\circ} \mathrm{C}$. The mixture was poured into crushed ice and the title compound 1,2,4-triazole was collected after being recrystallized.

1-(3,4-Dichlorobenzyl)-3-methoxy-4-methyl-1H-1,2,4-triazol5(4H)-one (5a). m.p. ${ }^{136}-140^{\circ} \mathrm{C}$, Yield $88 \%,{ }^{1} \mathrm{H} \mathrm{NMR}$ $\left(400 \mathrm{M}, \mathrm{CDCl}_{3}\right): 3.16$ (s, 3H, N-CH$\left.)_{3}\right), 3.96$ (s, 3H, $\mathrm{OCH}_{3}$ ), 4.83 (s, 2H, $\left.\mathrm{NCH}_{2}\right), 7.18-7.21(\mathrm{~m}, 1 \mathrm{H}, \mathrm{Ph}), 7.40-7.45(\mathrm{~m}$, $2 \mathrm{H}, \mathrm{Ph}) ; \mathrm{IR} / \mathrm{cm}^{-1}: 3449.33,2957.94,1710.43,1615.33,1518.36$, $1470.76,1425.27,1403.40,1307.42,1233.371135 .45,1009.21$, 914.98, 812.11, 743.63, 662.08, 595.11; ESI-MS: $289[\mathrm{M}+\mathrm{H}]^{+}$. Elemental anal. (\%), calculated: C, 45.85; H, 3.85; N, 14.58; found: C, 45.98; H, 3.77; N, 14.43.

1-(4-Bromobenzyl)-3-methoxy-4-methyl-1H-1,2,4-triazol5(4H)-one (5b). m.p. $138-140^{\circ} \mathrm{C}$, Yield $84 \%,{ }^{1} \mathrm{H}$ NMR (400 M, $\left.\mathrm{CDCl}_{3}\right): 3.15\left(\mathrm{~s}, 3 \mathrm{H}, \mathrm{N}-\mathrm{CH}_{3}\right), 3.95\left(\mathrm{~s}, 3 \mathrm{H}, \mathrm{OCH}_{3}\right), 4.84$ (s,
2H, $\mathrm{NCH}_{2}$ ), 7.24 (d, $\left.J=6.4 \mathrm{~Hz}, 2 \mathrm{H}, \mathrm{Ph}\right), 7.47$ (d, $J=6.4 \mathrm{~Hz}$, $2 \mathrm{H}, \mathrm{Ph}) ; \mathrm{IR} / \mathrm{cm}^{-1}: 3450.50,2942.58,1711.13,1607.26,145.83$, $1424.33,1382.18,1301.13,1067.47,1008.00$, 910.76, 847.46, 798.29, 727.42, 600.46; ESI-MS: $299[\mathrm{M}+\mathrm{H}]^{+}$. Elemental anal. (\%), calculated: C, 44.31; H, 4.06; N, 14.09; found: C, 44.25; H, 3.92; N, 14.21.

1-(2,4-Dichlorobenzyl)-3-methoxy-4-methyl-1H-1,2,4-triazol5(4H)-one (5c). m.p. $113-115^{\circ} \mathrm{C}$, Yield 89\%, ${ }^{1} \mathrm{H}$ NMR (400 M, $\mathrm{CDCl}_{3}$ ): $3.17\left(\mathrm{~s}, 3 \mathrm{H}, \mathrm{N}-\mathrm{CH}_{3}\right), 3.97\left(\mathrm{~s}, 3 \mathrm{H}, \mathrm{OCH}_{3}\right), 4.99$ (s, $\left.2 \mathrm{H}, \mathrm{NCH}_{2}\right), 7.24(\mathrm{~d}, J=8.4 \mathrm{~Hz}, 1 \mathrm{H}, \mathrm{Ph}), 7.22(\mathrm{~d}, J=8.4 \mathrm{~Hz}$, $1 \mathrm{H}, \mathrm{Ph}), 7.41$ (s, $1 \mathrm{H}, \mathrm{Ph}) ; \mathrm{IR} / \mathrm{cm}^{-1}: 3440.43,2943.89,1710.82$, $1606.45,1522.24,1490.26,1455.07,1424.03,1382.14,1318.96$, 1229.32, 1091.39, 1006.22, 849.44, 803.25, 599.99; ESI-MS: 289 $[\mathrm{M}+\mathrm{H}]^{+}$. Elemental anal. (\%), calculated: C, 45.85; H, 3.85; $\mathrm{N}, 14.58$; found: C, 45.67; H, 3.77; N, 14.64 .

1-(4-Chlorobenzyl)-3-methoxy-4-methyl-1H-1,2,4-triazol5(4H)-one (5d). m.p. $125-127^{\circ} \mathrm{C}$, Yield 82\%, ${ }^{1} \mathrm{H}$ NMR (400 M, $\mathrm{CDCl}_{3}$ ): $3.15\left(\mathrm{~s}, 3 \mathrm{H}, \mathrm{N}-\mathrm{CH}_{3}\right), 3.95\left(\mathrm{~s}, 3 \mathrm{H}, \mathrm{OCH}_{3}\right), 4.80$ (s, $2 \mathrm{H}, \mathrm{NCH}_{2}$ ), 7.28-7.31 (m, 4H, Ph); IR/ $\mathrm{cm}^{-1}: 3430.91,2954.61$, $1723.39,1611.41,1517.41,1470.16,1414.48,1352.86,1302.39$, $1230.3,1050.80,1010.51,912.22,878.42,829.12,788.23,741.01$, 691.10, 592.31, 468.63; ESI-MS: $254[\mathrm{M}+\mathrm{H}]^{+}$. Elemental anal. (\%), calculated: C, 52.08; H, 4.77; N, 16.56; found: C, 51.89; $\mathrm{H}, 4.87 ; \mathrm{N}, 16.65$.

4-((3-Methoxy-4-methyl-5-oxo-4,5-dihydro-1H-1,2,4-triazol1-yl)methyl)benzonitrile (5e). m.p. $160-162^{\circ} \mathrm{C}$, Yield $89 \%$, ${ }^{1} \mathrm{H}$ NMR (400 M, $\left.\mathrm{CDCl}_{3}\right): 3.15\left(\mathrm{~s}, 3 \mathrm{H}, \mathrm{N}-\mathrm{CH}_{3}\right), 3.96$ (s, 3H, $\left.\mathrm{OCH}_{3}\right), 4.94\left(\mathrm{~s}, 2 \mathrm{H}, \mathrm{NCH}_{2}\right), 7.44(\mathrm{~d}, J=8.16 \mathrm{~Hz}, 2 \mathrm{H}, \mathrm{Ph})$, $7.64(\mathrm{~d}, J=8.16 \mathrm{~Hz}, 2 \mathrm{H}, \mathrm{Ph}) ; \mathrm{IR} / \mathrm{cm}^{-1}: 3434.79,2955.79$, 2229.46, 1715.92, 1613.56, 1523.56, 1421.90, 1230.01, 1016.35, 857.47, 735.86, 640.39, 596.36, 552.70; ESI-MS: $245[\mathrm{M}+\mathrm{H}]^{+}$. Elemental anal. (\%), calculated: C, 59.01; H, 4.95; N, 22.94; found: C, 58.88; H, 5.12; N, 23.13.

(E)-Methyl 2-(2-((3-Methoxy-4-methyl-5-oxo-4,5-dihydro-1H1,2,4-triazol-1-yl)methyl)phenyl)-2-(methoxyimino)acetate (5f). m.p. $103-107^{\circ} \mathrm{C}$, Yield 89\%, ${ }^{1} \mathrm{H}$ NMR (400 M, $\left.\mathrm{CDCl}_{3}\right): 3.15$ (s, $3 \mathrm{H}, \mathrm{N}-\mathrm{CH}_{3}$ ), 3.96 (s, 3H, $\mathrm{OCH}_{3}$ ), 4.94 (s, 2H, $\mathrm{NCH}_{2}$ ), 7.44 (d, $J=8.16 \mathrm{~Hz}, 2 \mathrm{H}, \mathrm{Ph}), 7.64(\mathrm{~d}, J=8.16 \mathrm{~Hz}, 2 \mathrm{H}, \mathrm{Ph}) ; \mathrm{IR} / \mathrm{cm}^{-1}$ : 3455.02, 2936.45, 1720.46, 1611.72, 1522.17, 1431.39, 1230.32, 1005.55, 86.57, 741.29, 711.75, 681.57, 598.40, 572.80; ESI-MS: $245[\mathrm{M}+\mathrm{H}]^{+}$. Elemental anal. (\%), calculated: C, 53.89; H, 5.43; N, 16.76; found: C, 53.98; H, 5.13; N, 16.88. 
3-Methoxy-4-methyl-1-undecyl-1H-1,2,4-triazol-5(4H)-one (5g). m.p. $102-105^{\circ} \mathrm{C}$, Yield $78 \%,{ }^{1} \mathrm{H} \mathrm{NMR} \mathrm{(400} \mathrm{M,} \mathrm{CDCl}_{3}$ ): $0.86\left(\mathrm{t}, J=6.71 \mathrm{~Hz}, 3 \mathrm{H}, \mathrm{CH}_{3}\right), 1.24-1.29\left(\mathrm{~m}, 16 \mathrm{H}, \mathrm{CH}_{2}\right), 1.66-$ $1.68\left(\mathrm{~m}, 2 \mathrm{H}, \mathrm{CH}_{2}\right.$ ), 3.10 (s, 3H, N-CH $\left.\mathrm{CH}_{3}\right), 3.66$ (s, $J=7.21 \mathrm{~Hz}$, $2 \mathrm{H}, \mathrm{NCH}_{2}$ ), 3.95 (s, 3H, $\mathrm{OCH}_{3}$ ); IR/ $\mathrm{cm}^{-1}$ : 3446.77, 2941.81, 1708.02, 1622.82, 1258.48, 1131.46, 1101.03, 763.78, 661.94; ESI-MS: $285[\mathrm{M}+\mathrm{H}]^{+}$. Elemental anal. (\%), calculated: C, 63.57; H, 10.31; N, 14.83; found: C, 63.76; H, 10.52; N, 14.97 .

1-(3-Chlorobenzyl)-3-methoxy-4-methyl-1H-1,2,4-triazol5(4H)-one (5h). m.p. $97-100^{\circ} \mathrm{C}$, Yield 90\%, ${ }^{1} \mathrm{H}$ NMR (400 M, $\mathrm{CDCl}_{3}$ ): $3.17\left(\mathrm{~s}, 3 \mathrm{H}, \mathrm{N}-\mathrm{CH}_{3}\right), 3.96\left(\mathrm{~s}, 3 \mathrm{H}, \mathrm{OCH}_{3}\right), 4.87$ (s, $2 \mathrm{H}$, $\left.\mathrm{NCH}_{2}\right), 7.27-7.34(\mathrm{~m}, 4 \mathrm{H}, \mathrm{Ph}) ; \mathrm{IR} / \mathrm{cm}^{-1}: 3441.67,2960.00$, $1716.14,1621.83,1520.80,1391.88,1267.10,1228.92,014.96$, 789.51, 740.97, 593.84; ESI-MS: $254[\mathrm{M}+\mathrm{H}]^{+}$. Elemental anal. (\%), calculated: C, 52.08; H, 4.77; N, 16.56; found: C, 52.21; H, 4.87; N, 16.77 .

1-(2-Chlorobenzyl)-3-methoxy-4-methyl-1H-1,2,4-triazol5(4H)-one (5i). m.p. $120-122^{\circ} \mathrm{C}$, Yield $87 \%,{ }^{1} \mathrm{H}$ NMR (400 M, $\mathrm{CDCl}_{3}$ ): $3.19\left(\mathrm{~s}, 3 \mathrm{H}, \mathrm{N}-\mathrm{CH}_{3}\right), 3.97$ (s, $\left.3 \mathrm{H}, \mathrm{OCH}_{3}\right), 5.04$ (s, $2 \mathrm{H}$, $\mathrm{NCH}_{2}$ ), 7.19-7.25 (m, 2H, Ph), 7.37-7.39 (m, 2H, Ph); IR/cm ${ }^{-1}$ : 3439.30, 2947.24, 1718.79, 1614.24, 1523.69, 745.45, 595.23; ESI-MS: $254[\mathrm{M}+\mathrm{H}]^{+}$. Elemental anal. (\%), calculated: C, 52.08; $\mathrm{H}, 4.77 ; \mathrm{N}, 16.56$; found: $\mathrm{C}, 52.22 ; \mathrm{H}, 4.88 ; \mathrm{N}, 6.67$.

3-((3-Methoxy-4-methyl-5-oxo-4,5-dihydro-1H-1,2,4-triazol1-yl)methyl)benzonitrile (5j). m.p. $130^{\circ} \mathrm{C}$, Yield $89 \%,{ }^{1} \mathrm{H}$ NMR $\left(400 \mathrm{M}, \mathrm{CDCl}_{3}\right): 3.17$ (s, 3H, N-CH $\left.\mathrm{CH}_{3}\right), 3.97$ (s, 3H, $\mathrm{OCH}_{3}$ ), $4.91\left(\mathrm{~s}, 2 \mathrm{H}, \mathrm{NCH}_{2}\right), 7.46-7.48(\mathrm{~m}, 1 \mathrm{H}, \mathrm{Ph}), 7.58-7.62(\mathrm{~m}, 3 \mathrm{H}$, $\mathrm{Ph}) ; \mathrm{IR} / \mathrm{cm}^{-1}: 3446.70,2946.55,2231.74,1706.17,1522.05$, 1383.55, 1229.24, 1007.72, 786.75, 743.50, 702.92; ESI-MS: 245 $[\mathrm{M}+\mathrm{H}]^{+}$. Elemental anal. (\%), calculated: C, 59.01; H, 4.95; N, 22.94; found: C, 58.88; H, 4.89; N, 22.78.

3-Methoxy-1-(4-methoxybenzyl)-4-methyl-1H-1,2,4-triazol5(4H)-one (5k). m.p. 88-90 ${ }^{\circ} \mathrm{C}$, Yield 93\%, ${ }^{1} \mathrm{H}$ NMR (400 M, $\mathrm{CDCl}_{3}$ ): 3.14 (s, 3H, N-CH$\left.)_{3}\right), 3.81\left(\mathrm{~s}, 3 \mathrm{H}, \mathrm{OCH}_{3}\right), 3.95$ (s, $3 \mathrm{H}$, $\left.\mathrm{OCH}_{3}\right), 4.82\left(\mathrm{~s}, 2 \mathrm{H}, \mathrm{NCH}_{2}\right), 6.87$ (d, $\left.J=8.14 \mathrm{~Hz}, 2 \mathrm{H}, \mathrm{Ph}\right), 7.31$ (d, $J=8.14 \mathrm{~Hz}, 2 \mathrm{H}, \mathrm{Ph}) ; \mathrm{IR} / \mathrm{cm}^{-1}: 3444.95,2924.39,1721.08$, 1612.50, 1523.71, 1434.36, 1230.54, 742.97, 598.46; ESI-MS: 250 $[\mathrm{M}+\mathrm{H}]^{+}$. Elemental anal. (\%), calculated: C, 57.82; H, 6.07; N, 16.86; found: C, 58.01; H, 6.21; N, 16.76.

1-(3-Fluorobenzyl)-3-methoxy-4-methyl-1H-1,2,4-triazol5(4H)-one (5l). m.p. $102-103^{\circ} \mathrm{C}$, Yield 87\%, ${ }^{1} \mathrm{H}$ NMR (400 M, $\left.\mathrm{CDCl}_{3}\right): 3.14\left(\mathrm{~s}, 3 \mathrm{H}, \mathrm{N}-\mathrm{CH}_{3}\right), 3.94\left(\mathrm{~s}, 3 \mathrm{H}, \mathrm{OCH}_{3}\right), 4.86(\mathrm{~s}, 2 \mathrm{H}$, $\mathrm{NCH}_{2}$ ), 6.96-7.30 (m, 4H, Ph); IR/cm ${ }^{-1}: 3448.98,2936.21$, 1720.91, 1612.49, 1517.26, 1244.10, 1018.13, 595.24; ESI-MS: 238 $[\mathrm{M}+\mathrm{H}]^{+}$. Elemental anal. (\%), calculated: C, 55.69; H, 5.10; N, 17.71; found: C, 55.79; H, 5.12; N, 17.77.

1-(4-Fluorobenzyl)-3-methoxy-4-methyl-1H-1,2,4-triazol5(4H)-one (5m). m.p. 102-103 ${ }^{\circ} \mathrm{C}$, Yield 91\%, ${ }^{1} \mathrm{H}$ NMR (400 M, $\mathrm{CDCl}_{3}$ ): 3.15 (s, 3H, N-CH ), 3.95 (s, 3H, $\left.\mathrm{OCH}_{3}\right), 4.97$ (s, 2H, $\mathrm{NCH}_{2}$ ), 7.05-7.12 (m, 2H, Ph), 7.27-7.29 (m, 2H, Ph); $\mathrm{IR} / \mathrm{cm}^{-1}: 3427.88,2956.26,1709.34,1611.3,1519.26,1453.60$, $1399.75,1235.67,1134.51,1012.75,783.65,745.36,700.77$; ESI-MS: $238[\mathrm{M}+\mathrm{H}]^{+}$. Elemental anal. (\%), calculated: C, 55.69; H, 5.10; N, 17.71; found: C, 55.76; H, 5.22; N, 17.89 .
1-((5-Chloropyridin-2-yl)methyl)-3-methoxy-4-methyl-1H-1,2, 4-triazol-5(4H)-one (5n). m.p. $111-113^{\circ} \mathrm{C}$, Yield $88 \%,{ }^{1} \mathrm{H}$ $\mathrm{NMR}\left(400 \mathrm{M}, \mathrm{CDCl}_{3}\right): 3.15\left(\mathrm{~s}, 3 \mathrm{H}, \mathrm{N}-\mathrm{CH}_{3}\right), 3.95(\mathrm{~s}, 3 \mathrm{H}$, $\left.\mathrm{OCH}_{3}\right), 4.48\left(\mathrm{~s}, 2 \mathrm{H}, \mathrm{NCH}_{2}\right), 7.30-7.33(\mathrm{~m}, 1 \mathrm{H}, \mathrm{Py}), 7.68(\mathrm{~d}, J=$ $8.0 \mathrm{~Hz}, 1 \mathrm{H}, \mathrm{Py}), 8.42$ (s, 1H, Py); IR/ $\mathrm{cm}^{-1}:$ 3436.68, 2955.93, $1711.34,1611.26,1519.05,1399.59,1235.83,1134.81,1012.73$, 783.64, 701.50; ESI-MS: $256[\mathrm{M}+\mathrm{H}]^{+}$. Elemental anal. (\%), calculated: C, 47.16; H, 4.35; N, 22.00; found: C, 47.32; H, $4.44 ; \mathrm{N}, 22.12$.

1-(2-Fluorobenzyl)-3-methoxy-4-methyl-1H-1,2,4-triazol5(4H)-one (5o). m.p. 103-104 ${ }^{\circ} \mathrm{C}$, Yield 91\%, ${ }^{1} \mathrm{H}$ NMR (400 M, $\left.\mathrm{CDCl}_{3}\right): 3.19\left(\mathrm{~s}, 3 \mathrm{H}, \mathrm{N}-\mathrm{CH}_{3}\right), 3.97\left(\mathrm{~s}, 3 \mathrm{H}, \mathrm{OCH}_{3}\right), 5.04(\mathrm{~s}$, $2 \mathrm{H}, \mathrm{NCH}_{2}$ ), 7.19-7.22 (m, 1H, Ph), 7.24-7.28 (m, 2H, Ph), 7.37-7.39 (m, 1H, Ph); IR/cm ${ }^{-1}$ : 3436.50, 2934.06, 1716.72, $1613.09,1521.04,1416.95,1299.19,1230.77,1106.39,1004.22$, 826.93, 595.53; ESI-MS: $238[\mathrm{M}+\mathrm{H}]^{+}$. Elemental anal. (\%), calculated: C, 55.69; H, 5.10; N, 17.71; found: C, 55.47; H, 5.12; $\mathrm{N}, 17.88$.

3.3. Antifungal Activities. The biological activities of title compounds against Phytophthora infestans, Botrytis cinerea, Corynespora cassiicola, Rhizoctonia solani, and Pythium ultimum were evaluated according to [37-40], and a potted plant test method was adopted.

Germination was conducted by soaking cucumber seeds in water for $2 \mathrm{~h}$ at $50^{\circ} \mathrm{C}$ and then keeping the seeds moist for $24 \mathrm{~h}$ at $28^{\circ} \mathrm{C}$ in an incubator. When the radicles were $0.5 \mathrm{~cm}$, the seeds were grown in plastic pots containing a $1: 1(\mathrm{v} / \mathrm{v})$ mixture of vermiculite and peat. Cucumber and tomato plants used for inoculations were at the stage of two seed leaves. Tested compounds and commercial fungicides were sprayed with a hand spray on the surface of the seed leaves on a fine morning at the standard concentration of $500 \mu \mathrm{g} / \mathrm{mL}$; dimethomorph, fludioxonil, chlorothalonil, validamycin, and zhongshengmycin were used as control. After $2 \mathrm{~h}$, inoculation of Phytophthora infestans was carried out by spraying fungal suspension, inoculation of Rhizoctonia solani and Corynespora cassiicola was carried out by spraying mycelial suspension, and inoculation of Botrytis cinerea was carried out by radicle soaking. Pythium ultimum was found in the cucumber in nature. The experiment was repeated 4 times. After inoculation, the plants were maintained at 18 $30^{\circ} \mathrm{C}$ (mean temperature of $24^{\circ} \mathrm{C}$ and above $80 \%$ relative humidity $(\mathrm{RH})$ ). The fungicidal activity was evaluated when the nontreated cucumber plant (blank) fully developed symptoms. The area of inoculated treated leaves covered by disease symptoms was assessed and compared to that of nontreated ones to determine the average disease index. The relative control efficacy of compounds compared to the blank assay was calculated via the following equation:

$$
\text { relative control efficacy }(\%)=\frac{(\mathrm{CK}-\mathrm{PT})}{\mathrm{CK}} \times 100 \% \text {, }
$$

where CK is the average disease index during the blank assay and PT is the average disease index after treatment during testing.

3.4. DFT Calculation. DFT-B3LYP/6-31G (d) methods in Gaussian 03 package [41] were used to optimize the structure 
of 5c. Vibration analysis showed that the optimized structures were in accordance with the minimum points on the potential energy surfaces. All the convergent precisions were the system default values, and all the calculations were carried out on the DELL personal computer.

\section{Conclusion}

In summary, this paper reported some novel 1,2,4-triazol$5(4 H)$-one derivatives were successfully synthesized. The bioassay results showed that some of the title compounds exhibited considerable antifungal activity. The bioactivity of these novel compounds deserves further investigation.

\section{Conflict of Interests}

The authors declare that they have no conflict of interests.

\section{Acknowledgments}

The paper was supported by National Natural Science Foundation (no. 31401691), National Natural Science Foundation of Zhejiang Province (no. LQ13B020003), and Applied Chemistry Foundation of Zhejiang Shuren University (YH2013X14, YH2012S09). The authors also thank Dr. Xing-Hai Liu for assistance in this paper. The authors thank Professor Li Bao-Ju and Dr. Yan-Xia Shi for determining the antifungal activities.

\section{References}

[1] M. S. Mohamed, S. A. Ali, D. H. A. Abdelaziz, and S. S. Fathallah, "Synthesis and evaluation of novel pyrroles and pyrrolopyrimidines as anti-hyperglycemic agents," BioMed Research International, vol. 2014, Article ID 249780, 13 pages, 2014.

[2] S.-L. Yan, M.-Y. Yang, Z.-H. Sun et al., "Synthesis and antifungal activity of 1,2,3-thiadiazole derivatives containing 1,3,4thiadiazole moiety," Letters in Drug Design \& Discovery, vol. 11, no. 7, pp. 940-943, 2014.

[3] N. M. Khalifa and M. A. Al-Omar, "Synthesis and biological evaluation of 2-thioxopyrimidin-4(1H)-one derivatives as potential non-nucleoside HIV-1 reverse transcriptase inhibitors," International Journal of Molecular Sciences, vol. 15, no. 11, pp. 20723-20735, 2014.

[4] M.-Y. Yang, W. Zhao, Z.-H. Sun, C.-X. Tan, J.-Q. Weng, and X.H. Liu, "Synthesis and biological activity of acylthiourea derivatives contain 1,2,3-thiadiazole and 1,3,4-thiadiazole," Letters in Drug Design \& Discovery, vol. 12, no. 4, pp. 314-318, 2015.

[5] S. Bala, S. Kamboj, A. Kajal, V. Saini, and D. N. Prasad, "1,3,4Oxadiazole derivatives: synthesis, characterization, antimicrobial potential, and computational studies," BioMed Research International, vol. 2014, Article ID 172791, 18 pages, 2014.

[6] N.-N. Su, Y. Li, S.-J. Yu, X. Zhang, X.-H. Liu, and W.-G. Zhao, "Microwave-assisted synthesis of some novel 1,2,3-triazoles by click chemistry, and their biological activity," Research on Chemical Intermediates, vol. 39, no. 2, pp. 759-766, 2013.

[7] G.-X. Sun, Z.-H. Sun, M.-Y. Yang, X.-H. Liu, Y. Ma, and Y.Y. Wei, "Design, synthesis, biological activities and 3D-QSAR of new N,N'-diacylhydrazines containing 2,4-dichlorophenoxy moieties," Molecules, vol. 18, no. 12, pp. 14876-14891, 2013.
[8] X. H. Liu, X. Y. Xu, C. X. Tan, J. Q. Weng, J. H. Xin, and J. Chen, "Synthesis, crystal structure, herbicidal activities and 3D-QSAR study of some novel 1,2,4-triazolo[4,3-a]pyridine derivatives," Pest Management Science, vol. 71, no. 2, pp. 292-301, 2015.

[9] L.-J. Zhang, M.-Y. Yang, Z.-H. Sun et al., "Synthesis and antifungal activity of 1,3,4-thiadiazole derivatives containing pyridine group," Letters in Drug Design \& Discovery, vol. 11, no. 9, pp. 1107-1111, 2014.

[10] A. Parate and S. C. Chaturvedi, "Structural insights for 3H-1, 2, -4 triazolinones as angiotensin II receptor antagonists using QSAR techniques," Medicinal Chemistry Research, vol. 19, no. 4, pp. 375-391, 2010.

[11] S. K. Sivan and V. Manga, "Molecular docking and 3D-QSAR studies on triazolinone and pyridazinone, non-nucleoside inhibitor of HIV-1 reverse transcriptase," Journal of Molecular Modeling, vol. 16, no. 6, pp. 1169-1178, 2010.

[12] M. C. Sharma, S. Sharma, P. Sharma, A. Kumar, and K. S. Bhadoriya, "Comparative QSAR and pharmacophore analysis for a series of 2,4-dihydro-3H-1,2,4-triazol-3-ones derivatives as angiotensin II $\mathrm{AT}_{1}$ receptor antagonists," Medicinal Chemistry Research, vol. 23, no. 5, pp. 2486-2502, 2014.

[13] B. Côté, J. D. Burch, E. Asante-Appiah et al., "Discovery of MK1439 , an orally bioavailable non-nucleoside reverse transcriptase inhibitor potent against a wide range of resistant mutant HIV viruses," Bioorganic and Medicinal Chemistry Letters, vol. 24, no. 3, pp. 917-922, 2014.

[14] Z. K. Sweeney, S. Acharya, A. Briggs et al., "Discovery of triazolinone non-nucleoside inhibitors of HIV reverse transcriptase," Bioorganic and Medicinal Chemistry Letters, vol. 18, no. 15, pp. 4348-4351, 2008.

[15] Z. Liu, L. Pan, Y.-H. Li, S.-H. Wang, and Z.-M. Li, "Synthesis and herbicidal activity of novel sulfonylureas containing 1,2,4triazolinone moiety," Chemical Research in Chinese Universities, vol. 29, no. 3, pp. 466-472, 2013.

[16] L. Pan, Y. W. Chen, Z. Liu, Y. H. Li, and Z. M. Li, "Synthesis, crystal structure and herbicidal activity of novel sulfonylureas containing triazolinone moiety," Chinese Journal of Organic Chemistry, vol. 33, no. 3, pp. 542-550, 2013.

[17] Z. Yang, Y. Sheng-Gang, L. Yan-Ping et al., "Design and synthesis of 1-(benzothiazol-5-yl)-1H-1,2,4-triazol-5-ones as protoporphyrinogen oxidase inhibitors," Bioorganic \& Medicinal Chemistry, vol. 21, no. 11, pp. 3245-3255, 2013.

[18] O. G. Kol, H. Yuksek, and F. Islamoglu, "Synthesis and in vitro antioxidant activities of novel 4-(3-methyl-2thienylmethyleneamino)-4,5-dihydro-1H-1,2,4-triazol-5one derivatives with their acidic properties," Journal of the Chemical Society of Pakistan, vol. 35, no. 4, pp. 1179-1190, 2013.

[19] H. Yüksek, O. Akyildirim, M. L. Yola, Ö. Gürsoy-Kol, M. Çelebier, and D. Kart, "Synthesis, in vitro antimicrobial and antioxidant activities of some new 4,5-dihydro-1h-1,2,4-triazol5-one derivatives," Archiv der Pharmazie, vol. 346, no. 6, pp. 470-480, 2013.

[20] H. Yuksek, O. Akyildirim, and O. G. Kol, "Synthesis and in vitro antioxidant evaluation of new 1,3,5-Tri-2-methoxy4-[(4,5-dihydro- $1 H$-1,2,4-triazol-5-on -4-yl)-azomethine]phenoxycarbonyl-benzene derivatives," Journal of Chemistry, vol. 2013, Article ID 517420, 8 pages, 2013.

[21] M. Sharma, S. Garigipati, B. Kundu et al., "Discovery of novel 1,2,4-triazol-5-ones as tumor necrosis factor-alpha inhibitors for the treatment of neuropathic pain," Chemical Biology and Drug Design, vol. 80, no. 6, pp. 961-970, 2012. 
[22] M. Pitucha and J. Rzymowska, "Anticancer screening and structure activity relationship study of some semicarbazides and 1,2,4-Triazolin-5-ones," Letters in Drug Design and Discovery, vol. 9, no. 6, pp. 568-572, 2012.

[23] R. J. Singh and D. K. Singh, "Reaction of 4-amino-4,5-dihydro1H-1,2,4-triazol-5-one with some carboxylic acid anhydrides and their antiinflammatory activity," Asian Journal of Chemistry, vol. 22, no. 4, pp. 2664-2668, 2010.

[24] X.-H. Liu, J.-Q. Weng, B.-L. Wang, Y.-H. Li, C.-X. Tan, and Z.-M. Li, "Microwave-assisted synthesis of novel fluorinated 1,2,4-triazole derivatives, and study of their biological activity," Research on Chemical Intermediates, vol. 40, no. 8, pp. 26052612, 2014.

[25] G.-X. Sun, M.-Y. Yang, Y.-X. Shi et al., "Microwave assistant synthesis, antifungal activity and DFT theoretical study of some novel 1,2,4-triazole derivatives containing pyridine moiety," International Journal of Molecular Sciences, vol. 15, no. 5, pp. 8075-8090, 2014.

[26] X.-H. Liu, Z.-H. Sun, M.-Y. Yang et al., "Microwave assistant one pot synthesis, crystal structure, antifungal activities and 3D-QSAR of Novel 1,2,4-triazolo[4,3-a]pyridines," Chemical Biology \& Drug Design, vol. 84, no. 3, pp. 342-347, 2014.

[27] Z. H. Sun, Z. W. Zai, M. Y. Yang, X.-H. Liu, C.-X. Tan, and J.-Q. Weng, "Microwave assistant synthesis and dimeric crystal structure of 2-(((6-chloropyridin-3-yl)methyl)thio)-5(pyridin-4-yl)-1,3,4-thiadiazole," Chinese Journal of Structural Chemistry, vol. 33, pp. 1779-1783, 2014.

[28] L. J. Zhang, M. Y. Yang, B. Z. Hu et al., "Microwave assisted synthesis of novel 8-chloro-[1,2,4]triazolo[4,3-a]pyridine derivatives," Turkish Journal of Chemistry, 2015.

[29] N.-B. Sun, Y.-X. Shi, X.-H. Liu et al., "Design, synthesis, antifungal activities and 3D-QSAR of new $N, N^{\prime}$-diacylhydrazines containing 2,4-dichlorophenoxy moiety," International Journal of Molecular Sciences, vol. 14, no. 11, pp. 21741-21756, 2013.

[30] W. Ke, N.-B. Sun, and H.-K. Wu, "Microwave assistant synthesis, crystal structure and biological activity of A 1,2,4-triazole compound," Journal of the Chemical Society of Pakistan, vol. 35, no. 4, pp. 1239-1244, 2013.

[31] J. Y. Tong, H. K. Wu, N. B. Sun, and X. H. Liu, "Synthesis, crystal structure and biological activity of a new 1,2,4-triazole derivative," Chinese Journal of Structural Chemistry, vol. 32, pp. 607-611, 2013.

[32] N. B. Sun, X. H. Liu, J. Q. Weng, and C. X. Tan, "An unexpected product N-(3-((2-fluorobenzyl)thio)-5-methyl-4H-1,2,4triazol-4-yl)acetimidamide: synthesis and structure analysis," Journal of the Chemical Society of Pakistan, vol. 35, pp. 499-502, 2013.

[33] J.-Z. Jin and N.-B. Sun, "Synthesis, crystal structure and fungicidal activity of 3-(((4-Cyclopropyl-5-Methyl-4H-1,2,4-Triazol3-yl)Thio)Methyl)Benzonitrile $\mathrm{H}_{2} \mathrm{O}$ (1:1) solvent," Journal of the Chemical Society of Pakistan, vol. 35, no. 3, pp. 955-959, 2013.

[34] J.-C. Jin, Z.-H. Sun, M.-Y. Yang, J. Wu, and X.-H. Liu, "Synthesis, crystal structure, and theoretical studies of N(4((4-chlorobenzyl)oxy)phenyl)-4- (trifluoromethyl) pyrimidin2-amine," Journal of Chemistry, vol. 2013, Article ID 521757, 5 pages, 2013.

[35] Z.-J. Wang, Y. Gao, Y.-L. Hou et al., "Design, synthesis, and fungicidal evaluation of a series of novel 5-methyl-1H-1,2,3trizole-4-carboxyl amide and ester analogues," European Journal of Medicinal Chemistry, vol. 86, pp. 87-94, 2014.

[36] N.-B. Sun, J.-Q. Fu, J.-Q. Weng, J.-Z. Jin, C.-X. Tan, and X.H. Liu, "Microwave assisted synthesis, antifungal activity and
DFT theoretical study of some novel,1,2,4-triazole derivatives containing the 1,2,3-thiadiazole moiety," Molecules, vol. 18, no. 10, pp. 12725-12739, 2013.

[37] M. Y. Yang, W. Zhao, X.-H. Liu, J.-Q. Weng, and C.$\mathrm{X}$. Tan, "Synthesis, crystal structure and antifungal activity of 4-(5-((2,4-dichlorobenzyl)thio)-4-phenyl-4H-1,2,4-triazol3-yl)pyridine," Chinese Journal of Structural Chemistry, vol. 34, pp. 203-207, 2015.

[38] C.-X. Tan, Y.-X. Shi, J.-Q. Weng, X.-H. Liu, W.-G. Zhao, and B.-J. Li, "Synthesis and antifungal activity of novel 1,2,4triazole derivatives containing 1,2,3-thiadiazole moiety," Journal of Heterocyclic Chemistry, vol. 51, no. 3, pp. 690-694, 2014.

[39] L.-J. Min, C.-X. Tan, J.-Q. Weng, and X.-H. Liu, "Synthesis, crystal structure, and biological activity of a novel 1,2,3-thiadiazole compound containing 1,2,4-triazole moiety," Phosphorus, Sulfur and Silicon and the Related Elements, vol. 189, no. 3, pp. 379-386, 2014.

[40] G.-X. Sun, M.-Y. Yang, Z.-H. Sun, H.-K. Wu, X.-H. Liu, and Y.-Y. Wei, "Synthesis and bioactivities of novel 1,3,4-oxadiazole derivatives containing 1,2,3-thiadiazole moiety," Phosphorus, Sulfur, and Silicon and the Related Elements, vol. 189, no. 12, pp. 1895-1900, 2014.

[41] M. J. Frisch, G. W. Trucks, H. B. Schlegel et al., Gaussian 03, Revision C. 01, Gaussian, Wallingford, Conn, USA, 2004. 

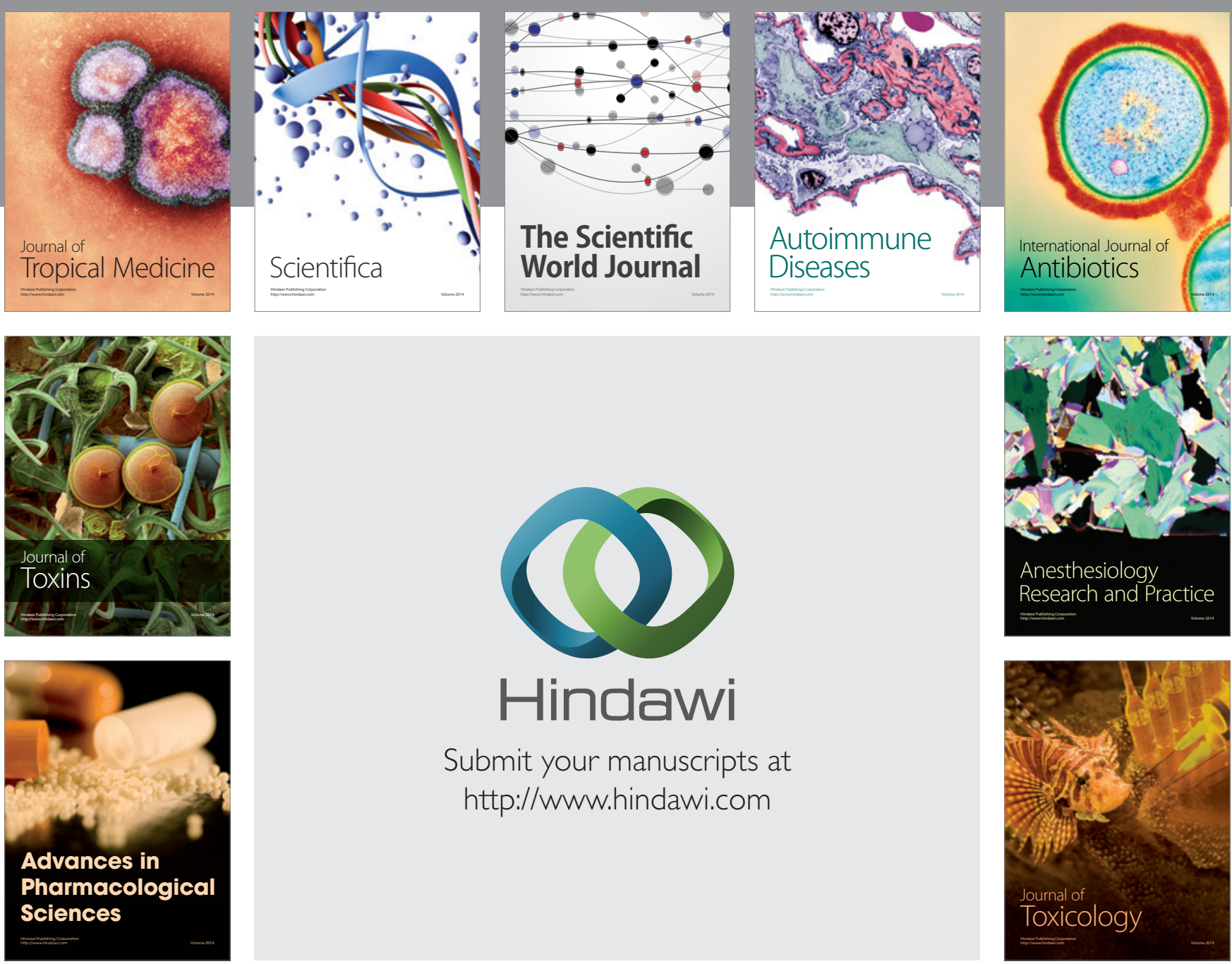

\section{Hindawi}

Submit your manuscripts at

http://www.hindawi.com
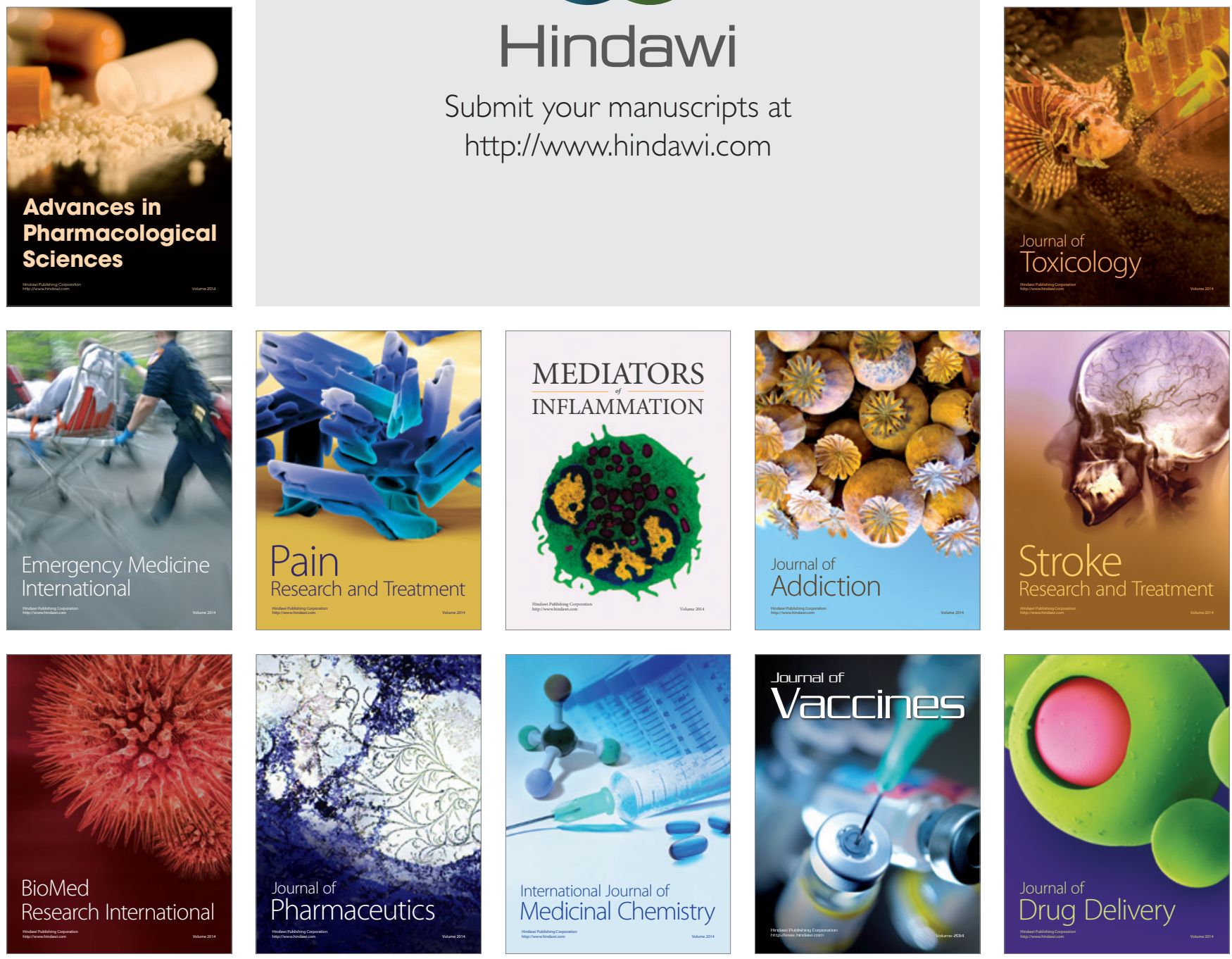